\title{
LOWER DIAMETER BOUNDS FOR COMPACT SHRINKING RICCI SOLITONS*
}

\author{
AKITO FUTAKI ${ }^{\dagger}$ AND YUJI SANO $\ddagger$
}

\begin{abstract}
It is shown that the diameter of a compact shrinking Ricci soliton has a universal lower bound. This is proved by extending universal estimates for the first non-zero eigenvalue of Laplacian on compact Riemannian manifolds with lower Ricci curvature bound to a twisted Laplacian on compact shrinking Ricci solitons.
\end{abstract}

Key words. Shrinking Ricci soliton, diameter bound.

AMS subject classifications. Primary 53C21; Secondary 53C20.

1. Introduction. In this paper we show that the diameter of compact shrinking Ricci solitons have a universal lower bound. Recall that Ricci solitons were introduced by Hamilton in [9] and are self-similar solutions to the Ricci flow. They are defined as follows.

Definition 1.1. A complete Riemannian metric $g$ on a smooth manifold $M^{n}$ is called a Ricci soliton if and only if there exist a constant $\gamma$ and a vector field $X$ such that

$$
2 \operatorname{Ric}(g)-2 \gamma g+\mathcal{L}_{X} g=0
$$

where $\operatorname{Ric}(g)$ and $\mathcal{L}_{X}$ respectively denote the Ricci tensor of $g$ and the Lie derivative along $X$. Moreover, if $X$ is the gradient vector field of a smooth function, $g$ is called a gradient Ricci soliton. The Ricci soliton is said to be shrinking, steady and expanding according as $\gamma>0, \gamma=0$ and $\gamma<0$.

If $X$ is zero, then $g$ is Einstein, in which case we say that $g$ is trivial. Due to Perelman (Remark 3.2, [17]), it is known that any Ricci soliton on a compact manifold is a gradient soliton. (See [5] for a direct Riemannian proof of Perelman's result.) It is also known that any nontrivial gradient Ricci soliton on a compact manifold is shrinking ([10], [11], see also [3]) with $n=\operatorname{dim} M \geq 4$. Examples of nontrivial compact KählerRicci solitons have been constructed by Koiso [12], Cao [2] and Wang and Zhu [18]. The main result of this paper is the following ${ }^{1}$.

THEOREM 1.2. Let $M^{n}$ be a compact smooth manifold with $n=\operatorname{dim} M \geq 4$. If $g$ is a non-trivial gradient shrinking Ricci soliton on $M$ satisfying (1), then

$$
d_{g} \geq \frac{10 \pi}{13 \sqrt{\gamma}}
$$

where $d_{g}$ is the diameter of $M$ with respect to $g$.

\footnotetext{
* Received November 4, 2011; accepted for publication February 23, 2012.

† Graduate School of Mathematical Sciences, University of Tokyo, 3-8-1 Komaba Meguro-ku Tokyo 153-8914, Japan (afutaki@ms.u-tokyo.ac.jp). The first author is supported by MEXT, Grant-in-Aid for Scientific Research (A), No. 21244003 and Challenging Exploratory Research No. 20654007.

${ }^{\ddagger}$ Graduate School of Science and Technology, Kumamoto University, 2-39-1, Kurokami, Kumamoto 860-8555, Japan (sano@sci.kumamoto-u.ac.jp). The second author is supported by MEXT, Grant-in-Aid for Young Scientists (B), No. 22740041.

${ }^{1}$ After the first version of this article was released on the ArXiv (July 2010), the estimate (2) is improved in [1] and [8].
} 
We may compare this result with the case of Einstein metrics. Suppose in (1) $X=0$ so that $g$ is an Einstein metric satisfying $\operatorname{Ric}(g)=\gamma g$. Then by Myers' theorem we have

$$
d_{g} \leq \sqrt{\frac{n-1}{\gamma}} \pi .
$$

Thus for compact Einstein manifolds the diameter is bounded from above while for nontrivial compact gradient shrinking Ricci solitons the diameter is bounded from below by a universal constant. Remark that Myers' theorem for compact Ricci solitons is known in [4]. Theorem 1.2 also implies a gap result for gradient shrinking Ricci solitons on a compact Riemannian manifold, i.e., if $d_{g}$ is strictly less than $\frac{10 \pi}{13 \sqrt{\gamma}}$, then $g$ should be Einstein. Other different types of gap theorems for gradient shrinking Ricci solitons are known. For example, see [6] and [13].

A similar result holds for any compact Kähler-Einstein manifold with positive $\gamma$ from a different argument, which is pointed out to us by Claude LeBrun. Since $\operatorname{Ric}(g)=\gamma g>0$, the volume of the manifold must be less than that of a ball of radius $d_{g}$ in the $2 n$-dimensional Euclidean space. The latter is equal to $\left(\pi^{n} / n !\right) d_{g}^{2 n}$. On the other hand, since the Kähler class is $(2 \pi / \gamma) c_{1}(M)$, the volume is $(2 \pi / \gamma)^{n} c_{1}(M)^{n}$. Since $c_{1}(M)^{n}$ is a positive integer, the volume is greater than $(2 \pi / \gamma)^{n}$. Combining these two estimates, we have

$$
d_{g} \geq \sqrt{\frac{2}{\gamma}}
$$

Remark that (4) is slightly weaker than (2), because $10 \pi / 13>2.4>\sqrt{2}$.

The proof of Theorem 1.2 is given by two steps. Assume that $g$ is a gradient shrinking soliton with respect to a gradient vector field $X$. Let $f \in C^{\infty}(M)$ be the potential function of $X$. Then, the equation (1) is equivalent to

$$
R_{i j}-\gamma g_{i j}+\nabla_{i} \nabla_{j} f=0
$$

where $R_{i j}$ denotes the Ricci curvature. Since $\gamma$ is positive, $\operatorname{Ric}(g)+\operatorname{Hess}(f)$ is positive definite. This means that Bakry-Émery geometry works on our case. Let $\Delta_{f}$ be the corresponding Bakry-Émery Laplacian, which is defined by

$$
\Delta_{f}=\Delta-\nabla f \cdot \nabla
$$

where $\Delta=g^{i j} \nabla_{i} \nabla_{j}$. We normalize $f$ so that it satisfies

$$
\int_{M} f e^{-f} d V_{g}=0
$$

The first step of the proof of Theorem 1.2 is to show that $-2 \gamma$ is an eigenvalue for $\Delta_{f}$. The second step is then to show that if $-\lambda$ is the first non-zero eigenvalue of $\Delta_{f}$ then $\lambda$ is bounded below by a universal constant:

$$
\lambda \geq \frac{\pi^{2}}{d_{g}^{2}}+\frac{31}{100} \gamma
$$

for $n \geq 3$. This estimate is an extension of a result of Ling [15] in the case of ordinary Laplacian to the case of Bakry-Émery Laplacian. A similar type of extension has 
been found by $\mathrm{Lu}$ and Rowlett (the first version of [16]) who extended a result of $\mathrm{Li}$ and Yau [14] in the case of ordinary Laplacian to the case of Bakry-Émery Laplacian.

This paper is organized as follows. In section 2 we show that that $-2 \gamma$ is an eigenvalue for $\Delta_{f}$. In section 3 we give a proof of (5). In section 4 we prove Theorem 1.2 .

2. An eigenfunction for the twisted Laplacian. Let $M$ be an $n$-dimensional compact Riemannian manifold and $g$ be a gradient shrinking soliton on $M$ so that we have

$$
R_{i j}-\gamma g_{i j}+\nabla_{i} \nabla_{j} f=0
$$

with $\gamma$ positive, and thus $\operatorname{Ric}(g)+\operatorname{Hess}(f)$ is positive definite. Let $\Delta_{f}$ be the corresponding Bakry-Émery Laplacian defined by

$$
\Delta_{f}=\Delta-\nabla f \cdot \nabla
$$

where $\Delta=g^{i j} \nabla_{i} \nabla_{j}$. We normalize $f$ so that it satisfies

$$
\int_{M} f e^{-f} d V_{g}=0
$$

Lemma 2.1. The function $f$ is an eigenfunction of $\Delta_{f}$ with eigenvalue equal to $-2 \gamma$.

Proof. Taking the covariant derivative of the left hand in (6) we have

$$
\begin{aligned}
0=\nabla_{k}\left(R_{i j}-\gamma g_{i j}+\nabla_{i} \nabla_{j} f\right) & =\nabla_{k} R_{i j}+\nabla_{i} \nabla_{k} \nabla_{j} f-R_{k i}{ }_{j} \nabla_{l} f \\
& =\nabla_{k} R_{i j}-\nabla_{i} R_{k j}-R_{k i}{ }_{j} \nabla_{l} f .
\end{aligned}
$$

Taking the trace of the above on $i$ and $j$,

$$
\nabla_{k} R-\nabla_{i} R_{k}^{i}-R_{k}^{l} \nabla_{l} f=0,
$$

where $R$ is the scalar curvature of $g$. Then this and the contracted second Bianchi identity

$$
\nabla_{i} R_{k}^{i}=\frac{1}{2} \nabla_{k} R
$$

induce

$$
\nabla_{k} R=2 R_{k}^{l} \nabla_{l} f
$$

From (6) and (8), we get

$$
\begin{aligned}
\nabla_{k}\left(R-2 \gamma f+|\nabla f|^{2}\right) & =2 R_{k}{ }^{l} \nabla_{l} f-2 \gamma \nabla_{k} f+2 \nabla_{k} \nabla_{l} f \nabla^{l} f \\
& =2\left(R_{k l}-\gamma g_{k l}+\nabla_{k} \nabla_{l} f\right) \nabla^{l} f \\
& =0 .
\end{aligned}
$$

Hence, there exists some constant $C$ such that

$$
R-2 \gamma f+|\nabla f|^{2}=C .
$$


Taking the trace of (6) on $i$ and $j$, we have

$$
R-n \gamma+\Delta f=0
$$

From (9) and (10), we have

$$
\Delta_{f} f=\Delta f-|\nabla f|^{2}=-2 \gamma f+C^{\prime}
$$

where $C^{\prime}$ is some constant. The equality (7) and

$$
\int_{M}\left(\Delta_{f} f\right) e^{-f} d V_{g}=0
$$

imply that the constant $C^{\prime}$ in (11) must be zero. The proof of the lemma is completed.

REMARK 2.2. Lemma 2.1 says that on a compact nontrivial gradient shrinking Ricci soliton, there always exists a eigenfunction of $\Delta_{f}$ with a fixed nonzero eigenvalue. A similar result holds for Fano manifolds, i.e. compact complex manifolds with positive first Chern class. If $M$ is such a manifold and $g$ is a Kähler metric whose Kähler form represents $c_{1}(M)$ then there exists a smooth function $f$ such that

$$
R_{i \bar{j}}=g_{i \bar{j}}+\partial_{i} \bar{\partial}_{j} f \text {. }
$$

It is shown in [7] that if $M$ has non-zero holomorphic vector fields then $\Delta_{f}$ has eigenfunctions with eigenvalue equal to -1 . The gradient vector fields of the eigenfunctions are the holomorphic vector fields.

3. Eigenvalue estimates for Bakry-Émery Laplacian. In this section, we extend Theorem 1 in [15] to Bakry-Émery geometry. We shall state the main result of this section in the end of this section (see Theorem 3.9).

Let $(M, g, \phi)$ be a Bakry-Émery manifold, that is to say, a triple of a Riemannian manifold $M$ with a Riemannian metric $g$ and a weighted volume form $e^{-\phi} d V_{g}$. The Bakry-Émery Ricci curvature is defined by

$$
\operatorname{Ric}_{\phi}:=\operatorname{Ric}(g)+\operatorname{Hess}(\phi)
$$

and the Bakry-Émery Laplacian is defined by

$$
\Delta_{\phi}:=\Delta-\nabla \phi \cdot \nabla
$$

where $\operatorname{Ric}(g)$ denotes the Ricci tensor of $g$ and $\operatorname{Hess}(\phi)$ the Hessian of $\phi$. Here we assume that $M$ is compact and has no boundary. Also we assume that $\operatorname{Ric}_{\phi}$ is strictly positive as follows. For some constant $K>0$,

$$
\operatorname{Ric}_{\phi} \geq(n-1) K g
$$

for all $x \in M$.

Let $u$ be an eigenfunction of the first non-zero eigenvalue $-\lambda$, i.e., $\Delta_{\phi} u=-\lambda u$. We normalize $u$ as follows. If $-\min u>\max u$, we replace $u$ by $-u$. Otherwise, we keep $u$ unchanged. Then, denoting $u / \max u$ by the same letter $u$ again, we can assume that $u$ satisfies

$$
\max u=1, \min u=-k, 0<k \leq 1 \text {. }
$$


We define a function $v$ by

$$
v:=[u-(1-k) / 2][(1+k) / 2] .
$$

Then, $v$ satisfies

$$
\max v=1, \min v=-1,
$$

and

$$
\Delta_{\phi} v=-\lambda(v+a)
$$

where

$$
a:=(1-k) /(1+k) .
$$

Note that $0 \leq a<1$.

Proposition 3.1.

$$
\lambda \geq(n-1) K
$$

REMARK 3.2. This estimate is weaker than the one in [15].

Proof. At any $x \in M$, we have

$$
\begin{aligned}
\Delta\left(|\nabla v|^{2}\right)= & \nabla^{j} \nabla_{j}\left(\nabla_{i} v \nabla^{i} v\right) \\
= & 2 \nabla^{j}\left(\nabla_{j} \nabla_{i} v \nabla^{i} v\right) \\
= & 2 \nabla^{j} \nabla_{j} \nabla_{i} v \nabla^{i} v+2|\nabla \nabla v|^{2} \\
= & 2\left(\nabla_{i}(\Delta v) \nabla^{i} v+\operatorname{Ric}(\nabla v, \nabla v)\right)+2|\nabla \nabla v|^{2} \\
\geq & 2\left\{\nabla_{i}\left(-\lambda(v+a)+\nabla^{j} \phi \nabla_{j} v\right) \nabla^{i} v+(n-1) K|\nabla v|^{2}\right. \\
& \left.-\nabla_{i} \nabla_{j} \phi \nabla^{i} v \nabla^{j} v\right\}+2|\nabla \nabla v|^{2} \\
= & -2 \lambda|\nabla v|^{2}+2 \nabla^{i} v \nabla_{i} \nabla_{j} v \nabla^{j} \phi+2(n-1) K|\nabla v|^{2}+2|\nabla \nabla v|^{2} .
\end{aligned}
$$

We have

$$
\nabla^{j} \phi \nabla_{j}\left(|\nabla v|^{2}\right)=2 \nabla^{i} v \nabla_{i} \nabla_{j} v \nabla^{j} \phi
$$

Then, we have

$$
\frac{1}{2} \Delta_{\phi}\left(|\nabla v|^{2}\right) \geq((n-1) K-\lambda)|\nabla v|^{2}+|\nabla \nabla v|^{2} \geq((n-1) K-\lambda)|\nabla v|^{2} .
$$

Integrating both sides in the above inequality with respect to $e^{-\phi} d V_{g}$, we get the desired estimate. The proof is completed.

REMARK 3.3. In [15], the integral of $|\nabla \nabla v|^{2}$ over $M$ with respect to $d V_{g}$ is estimated by the integral of $|\nabla v|^{2}$, then it implies that $\lambda \geq n K$. However, the same way does not hold in the case of Bakry-Émery geometry. In fact, the error terms, which come from the gap between $\Delta$ and $\Delta_{\phi}$, are not cancelled.

Proposition 3.4. Let $b>1$ be an arbitrary constant. Then, $v$ satisfies

$$
\frac{|\nabla v|^{2}}{b^{2}-v^{2}} \leq \lambda(1+a) \text {. }
$$


Proof. Consider the function on $M$ defined by

$$
P(x):=|\nabla v|^{2}+A v^{2}, A:=\lambda(1+a)+\varepsilon
$$

for small $\varepsilon>0$. Let $x_{0} \in M$ be a point which $P$ attains its maximum. The maximum principle implies

$$
\nabla P\left(x_{0}\right)=0, \Delta P\left(x_{0}\right) \leq 0 .
$$

There are two cases, either $\nabla v\left(x_{0}\right)=0$ or $\nabla v\left(x_{0}\right) \neq 0$.

If $\nabla v\left(x_{0}\right)=0$, then

$$
|\nabla v|^{2}+A v^{2} \leq P\left(x_{0}\right)=A v^{2}\left(x_{0}\right) \leq A
$$

So

$$
\frac{|\nabla v|^{2}}{b^{2}-v^{2}} \leq \frac{1-v^{2}}{b^{2}-v^{2}} A \leq A
$$

Letting $\varepsilon \rightarrow 0$, we get (13).

If $\nabla v\left(x_{0}\right) \neq 0$, then we rotate the local orthonormal frame about $x_{0}$ such that

$$
\left|\nabla_{1} v\left(x_{0}\right)\right|=\left|\nabla v\left(x_{0}\right)\right| \neq 0, \quad \nabla_{i} v\left(x_{0}\right)=0 \text { for } i \geq 2 \text {. }
$$

Since

$$
\frac{1}{2} \nabla_{1} P\left(x_{0}\right)=\nabla^{1} v \nabla_{1} \nabla_{1} v+A v \nabla_{1} v=0
$$

we have

$$
\nabla_{1} \nabla_{1} v=-A v
$$

For $i \geq 2$, since

$$
\frac{1}{2} \nabla_{i} P\left(x_{0}\right)=\nabla^{j} v \nabla_{i} \nabla_{j} v+A v \nabla_{i} v=\nabla^{1} v \nabla_{i} \nabla_{1} v=0
$$

we have

$$
\nabla_{i} \nabla_{1} v=0
$$

From these two equalities, we get

$$
|\nabla \nabla v|^{2}=A^{2} v^{2}
$$

and

$$
\nabla^{j} v \nabla_{j} \nabla_{i} v=-A v \nabla_{i} v
$$

at $x_{0} \in M$. Then, at $x_{0} \in M$, we have

$$
\begin{aligned}
0 \geq & \frac{1}{2} \Delta P\left(x_{0}\right)=\frac{1}{2} \Delta\left(|\nabla v|^{2}+A v^{2}\right) \\
= & |\nabla \nabla v|^{2}+\nabla^{j} \nabla_{j} \nabla_{i} v \nabla^{i} v+A|\nabla v|^{2}+A v \Delta v \\
= & A^{2} v^{2}+\nabla^{j} \nabla_{j} \nabla_{i} v \nabla^{i} v+A|\nabla v|^{2}+A v \Delta v \quad(\text { due to }(16)) \\
= & A^{2} v^{2}+\nabla^{i} v\left(\nabla_{i}(\Delta v)\right)+\operatorname{Ric}(\nabla v, \nabla v)+A|\nabla v|^{2}+A v \Delta v \\
\geq & A^{2} v^{2}+\nabla^{i} v\left(\nabla_{i}\left(-\lambda(v+a)+\nabla_{j} \phi \nabla^{j} v\right)\right)+(n-1) K(\nabla v, \nabla v) \\
& -\nabla_{i} \nabla_{j} \phi \nabla^{i} v \nabla^{j} v+A|\nabla v|^{2}+A v\left(-\lambda(v+a)+\nabla_{i} \phi \nabla^{i} v\right) \\
= & A v^{2}-\lambda|\nabla v|^{2}+(n-1) K|\nabla v|^{2}+A|\nabla v|^{2}-A \lambda v^{2}-a A \lambda v \\
& (\text { due to }(17)) \\
\geq & (A-\lambda)|\nabla v|^{2}+A(A-\lambda) v^{2}-a A \lambda v .
\end{aligned}
$$


Then, at $x_{0} \in M$, we have

$$
\begin{aligned}
|\nabla v|^{2}+(A-\varepsilon) v^{2} & \leq-A v^{2}+\frac{a A \lambda v}{a \lambda+\varepsilon}+(A-\varepsilon) v^{2} \\
& =-\varepsilon v^{2}+\frac{a A \lambda v}{a \lambda+\varepsilon} .
\end{aligned}
$$

Letting $\varepsilon \rightarrow 0$, we get

$$
|\nabla v|^{2}(x)+A v^{2}(x) \leq A
$$

for any $x \in M$. Therefore, we can get the desired estimate as the former case. The proof is completed.

Define a function $Z$ by

$$
Z(t):=\max _{x \in U(t)} \frac{|\nabla v|^{2}}{\lambda\left(b^{2}-v^{2}\right)}
$$

where $U(t):=\left\{x \in M \mid \sin ^{-1}(v(x) / b)=t\right\}$. Note that

$$
t \in\left[-\sin ^{-1}(1 / b), \sin ^{-1}(1 / b)\right]
$$

Let

$$
c:=\frac{a}{b}, \alpha:=\frac{1}{2}(n-1) K, \delta:=\frac{\alpha}{\lambda} .
$$

Proposition 3.5. If the function $z:\left[-\sin ^{-1}(1 / b), \sin ^{-1}(1 / b)\right] \rightarrow \mathbb{R}$ satisfies the following

(a) $z(t) \geq Z(t)$ for all $t \in\left[-\sin ^{-1}(1 / b), \sin ^{-1}(1 / b)\right]$;

(b) there exists some $x_{0} \in M$ such that $z\left(t_{0}\right)=Z\left(t_{0}\right)$ at $t_{0}=\sin ^{-1}\left(v\left(x_{0}\right) / b\right)$;

(c) $z\left(t_{0}\right)>0$;

then we have

$$
\begin{aligned}
0 \leq & \frac{1}{2} \ddot{z}\left(t_{0}\right) \cos ^{2} t_{0}-\dot{z}\left(t_{0}\right) \cos t_{0} \sin t_{0}-z\left(t_{0}\right)+1+c \sin t_{0}-2 \delta \cos ^{2} t_{0} \\
& -\frac{\dot{z}\left(t_{0}\right)}{4 z\left(t_{0}\right)} \cos t_{0}\left\{\dot{z}\left(t_{0}\right) \cos t_{0}-2 z\left(t_{0}\right) \sin t_{0}+2 \sin t_{0}+2 c\right\}
\end{aligned}
$$

Proof. Define

$$
J(x):=\left\{\frac{|\nabla v|^{2}}{b^{2}-v^{2}}-\lambda z\right\} \cos ^{2} t
$$

where $t=\sin ^{-1}(v(x) / b)$. Since $z(t) \geq Z(t)$ and $z\left(t_{0}\right)=Z\left(t_{0}\right)$, we have

$$
J(x) \leq 0 \text { for } \forall x \in M, \quad J\left(x_{0}\right)=0 .
$$

Since $Z\left(t_{0}\right)>0$, we have

$$
\nabla v\left(x_{0}\right) \neq 0
$$


By the maximum principle, we have

$$
\nabla J\left(x_{0}\right)=0, \Delta J\left(x_{0}\right) \leq 0
$$

Since $\cos ^{2} t=1-(v / b)^{2}, J$ can be written by

$$
J(x)=\frac{1}{b^{2}}|\nabla v|^{2}-\lambda z \cos ^{2} t .
$$

Since $\nabla J\left(x_{0}\right)=0$, at $x_{0} \in M$

$$
\frac{2}{b^{2}} \nabla_{j} \nabla_{i} v \nabla^{i} v=\lambda \cos t\{\dot{z} \cos t-2 z \sin t\} \nabla_{j} t
$$

As before, we rotate the local orthonormal frame about $x_{0} \in M$ such that

$$
\left|\nabla_{1} v\left(x_{0}\right)\right|=\left|\nabla v\left(x_{0}\right)\right|
$$

and $\nabla_{i} v\left(x_{0}\right)=0$ for $i \geq 2$. Note that $\nabla_{i} t\left(x_{0}\right)=0$ for $i \geq 2$, because

$$
\nabla_{i} v\left(x_{0}\right)=b \cos t_{0} \nabla_{i} t\left(x_{0}\right)
$$

From (19) and (20), we have

$$
\nabla_{1} \nabla_{1} v=\frac{\lambda b}{2}(\dot{z} \cos t-2 z \sin t)
$$

and $\nabla_{j} \nabla_{1} v=0(j \geq 2)$ at $x_{0} \in M$.

Next we compute $\Delta J\left(x_{0}\right)$. At $x_{0} \in M$, we have

$$
\begin{aligned}
\Delta J\left(x_{0}\right)= & \Delta\left(\frac{1}{b^{2}}|\nabla v|^{2}-\lambda z \cos ^{2} t\right) \\
= & \frac{2}{b^{2}}|\nabla \nabla v|^{2}+\frac{2}{b^{2}} \nabla^{i} v\left(\nabla^{j} \nabla_{j} \nabla_{i} v\right) \\
& -\lambda \nabla^{j}\left(\dot{z} \cos ^{2} t \nabla_{j} t-2 z \cos t \sin t \nabla_{j} t\right) \\
= & \frac{2}{b^{2}}|\nabla \nabla v|^{2} \\
& +\frac{2}{b^{2}} \nabla^{i} v\left(\nabla^{j} \nabla_{j} \nabla_{i} v\right) \\
& -\lambda \ddot{z} \cos ^{2} t|\nabla t|^{2}-\lambda \dot{z} \cos ^{2} t(\Delta t) \\
& +4 \lambda \dot{z} \cos t \sin t|\nabla t|^{2}-\lambda z \Delta\left(\cos ^{2} t\right)
\end{aligned}
$$

Before estimating the above, we prepare some calculations. Remark that the following 
calculations are done at $x_{0} \in M$.

$$
\begin{aligned}
|\nabla v|^{2} & =\lambda b^{2} z \cos ^{2} t ; \quad\left(\text { due to } J\left(x_{0}\right)=0\right) \\
|\nabla t|^{2} & =\frac{|\nabla v|^{2}}{b^{2} \cos ^{2} t}=\lambda z ; \quad(\text { due to }(23)) \\
\frac{\Delta v}{b} & =\Delta \sin t=\cos t(\Delta t)-\sin t|\nabla t|^{2} ; \\
\Delta t & =\frac{1}{\cos t}\left(\sin t|\nabla t|^{2}+\frac{\Delta v}{b}\right) \quad(\text { due to }(25)) \\
& =\frac{1}{\cos t}\left(\lambda z \sin t-\frac{\lambda}{b}(v+a)+\frac{1}{b}\left(\nabla_{i} \phi \nabla^{i} v\right)\right) ; \quad(\text { due to }(24)) \\
\Delta \cos ^{2} t & =\Delta\left(1-\frac{v^{2}}{b^{2}}\right) \\
& =-\frac{2}{b^{2}}|\nabla v|^{2}-\frac{2}{b^{2}} v \Delta v \\
& =-2 \lambda z \cos ^{2} t-\frac{2}{b^{2}} v\left(-\lambda(v+a)+\nabla_{i} \phi \nabla^{i} v\right)
\end{aligned}
$$

Now, at $x_{0} \in M$ we get

$$
\begin{aligned}
\frac{2}{b^{2}}|\nabla \nabla v|^{2} & \geq \frac{2}{b^{2}}\left(\nabla_{1} \nabla_{1} v\right)^{2} \\
& =\frac{\lambda^{2}}{2}(\dot{z})^{2} \cos ^{2} t-2 \lambda^{2} z \dot{z} \cos t \sin t+2 \lambda^{2} z^{2} \sin ^{2} t, \quad(\text { due to }(21))
\end{aligned}
$$

and

$$
\begin{aligned}
\frac{2}{b^{2}} \nabla^{i} v\left(\nabla^{j} \nabla_{j} \nabla_{i} v\right)= & \frac{2}{b^{2}}\left(\nabla^{j} v \nabla_{j}(\Delta v)+\operatorname{Ric}(\nabla v, \nabla v)\right) \\
\geq & \frac{2}{b^{2}}\left(\nabla^{j} v \nabla_{j}(\Delta v)-\nabla_{i} \nabla_{j} \phi \nabla^{i} v \nabla^{j} v+2 \alpha|\nabla v|^{2}\right) \\
= & \frac{2}{b^{2}}\left(-\lambda|\nabla v|^{2}+\nabla_{i} \phi \nabla^{j} v \nabla_{j} \nabla^{i} v+2 \alpha|\nabla v|^{2}\right) \\
= & \left(-2 \lambda^{2}+4 \alpha \lambda\right) z \cos ^{2} t+\frac{2}{b^{2}} \nabla_{i} \phi \nabla^{j} v \nabla_{j} \nabla^{i} v, \\
& \text { (due to }(23))
\end{aligned}
$$

and

$$
\begin{aligned}
-\lambda\left(\ddot{z}|\nabla t|^{2}+\dot{z} \Delta t\right) \cos ^{2} t= & -\lambda^{2} z \ddot{z} \cos ^{2} t-\lambda \dot{z} \cos ^{2} t\left(\frac{1}{\cos t}[\lambda z \sin t\right. \\
& \left.\left.-\frac{\lambda}{b}(v+a)+\frac{1}{b}\left(\nabla_{i} \phi \nabla^{i} v\right)\right]\right) \quad(\text { due to }(24),(25)) \\
= & -\lambda^{2} z \ddot{z} \cos ^{2} t-\lambda^{2} z \dot{z} \cos t \sin t+\frac{1}{b} \lambda^{2} \dot{z}(v+a) \cos t \\
& -\frac{1}{b} \lambda \dot{z} \cos t \nabla_{i} \phi \nabla^{i} v
\end{aligned}
$$


and

$$
\begin{aligned}
4 \lambda \dot{z} \cos t \sin t|\nabla t|^{2}-\lambda z \Delta \cos ^{2} t= & 4 \lambda^{2} z \dot{z} \cos t \sin t-\lambda z\left(-2 \lambda z \cos ^{2} t\right. \\
& \left.+\frac{2}{b^{2}} \lambda v(v+a)-\frac{2}{b^{2}} v \nabla_{i} \phi \nabla^{i} v\right) \\
& (\text { due to }(24),(27)) \\
= & 4 \lambda^{2} z \dot{z} \cos t \sin t+2 \lambda^{2} z^{2} \cos ^{2} t \\
& -\frac{2}{b^{2}} \lambda^{2} z \sin t \cdot(v+a)+\frac{2}{b^{2}} \lambda z v \nabla_{i} \phi \nabla^{i} v
\end{aligned}
$$

Since (19) implies

$$
\begin{aligned}
\frac{2}{b^{2}} \nabla^{j} v \nabla_{j} \nabla^{i} v & =\lambda\left(\cos t \nabla^{i} t\right)[\dot{z} \cos t-2 z \sin t] \\
& =\frac{\lambda}{b} \nabla^{i} v[\dot{z} \cos t-2 z \sin t] \\
& =\frac{1}{b} \lambda \dot{z} \cos t \nabla^{i} v-\frac{2}{b^{2}} \lambda z v\left(\nabla^{i} v\right),
\end{aligned}
$$

getting $\Delta J\left(x_{0}\right) \leq 0,(22),(28),(29),(30)$ and (31) together, we have

$$
\begin{aligned}
0 \geq & -\lambda^{2} z \ddot{z} \cos ^{2} t+\frac{\lambda^{2}}{2}(\dot{z})^{2} \cos ^{2} t+\lambda^{2} \dot{z} \cos t(z \sin t+c+\sin t) \\
& +2 \lambda^{2} z^{2}-2 \lambda^{2} z-2 \lambda^{2} c z \sin t+4 \alpha \lambda z \cos ^{2} t
\end{aligned}
$$

at $x_{0} \in M$. Dividing the two sides in the above inequality by $2 \lambda^{2} z\left(x_{0}\right)>0$, we have

$$
\begin{aligned}
0 \geq & -\frac{1}{2} \ddot{z}\left(t_{0}\right) \cos ^{2} t_{0}+\frac{1}{2} \dot{z}\left(t_{0}\right) \cos t_{0}\left(\sin t_{0}+\frac{c+\sin t_{0}}{z\left(t_{0}\right)}\right)+z\left(t_{0}\right) \\
& -1-c \sin t_{0}+2 \delta \cos ^{2} t_{0}+\frac{1}{4 z\left(t_{0}\right)}\left(\dot{z}\left(t_{0}\right)\right)^{2} \cos ^{2} t_{0} .
\end{aligned}
$$

Therefore we get the desired inequality. The proof is completed.

Then, we have the same results as Corollary 6 and 7 in [15] from Proposition 3.5.

Corollary 3.6. Let the notations be as in Proposition 3.5.

(a) If $z(t)$ satisfies the conditions (a), (b), (c) in Proposition 3.5, $\dot{z}\left(t_{0}\right) \geq 0$ and $1-c \leq z\left(t_{0}\right) \leq 1+a$, then we have

(32) $0 \leq \frac{1}{2} \ddot{z}\left(t_{0}\right) \cos ^{2} t_{0}-\dot{z}\left(t_{0}\right) \cos t_{0} \sin t_{0}-z\left(t_{0}\right)+1+c \sin t_{0}-2 \delta \cos ^{2} t_{0}$.

(b) Suppose that $a=0$. If $z(t)$ satisfies the conditions (a), (b), (c) in Proposition 3.5, $\dot{z}\left(t_{0}\right) \sin t_{0} \geq 0$ and $z\left(t_{0}\right) \leq 1$, then we have

$$
0 \leq \frac{1}{2} \ddot{z}\left(t_{0}\right) \cos ^{2} t_{0}-\dot{z}\left(t_{0}\right) \cos t_{0} \sin t_{0}-z\left(t_{0}\right)+1-2 \delta \cos ^{2} t_{0}
$$

Proof. The proof is the same as [15], but we recall it for the reader's convenience. It is sufficient to show that the last term in (18) is nonnegative. First, let us prove 
the fist case (a). From the condition (c) in Proposition 3.5, $\dot{z}\left(t_{0}\right) \geq 0, \cos t_{0} \geq 0$ and $z\left(t_{0}\right)>0$, then it is sufficient to show that

$$
-z\left(t_{0}\right) \sin t_{0}+\sin t_{0}+c
$$

is nonnegative. When $t_{0} \geq 0$, we have

$$
-z\left(t_{0}\right) \sin t_{0}+\sin t_{0}+c \geq-(1+a) \sin t_{0}+\sin t_{0}+c \geq a\left(\frac{1}{b}-\sin t_{0}\right) \geq 0 .
$$

The last inequality in above follows from that $\left|\sin t_{0}\right|=\left|\nu\left(t_{0}\right) / b\right| \leq 1 / b$. When $t_{0}<0$, we have

$$
-z\left(t_{0}\right) \sin t_{0}+\sin t_{0}+c \geq-(1-c) \sin t_{0}+\sin t_{0}+c \geq c\left(1+\sin t_{0}\right) \geq 0 .
$$

Next we shall prove the second case (b). In this case, it is sufficient to show that

$$
\dot{z}\left(t_{0}\right)\left(-z\left(t_{0}\right) \sin t_{0}+\sin t_{0}\right)=\left(1-z\left(t_{0}\right)\right) \dot{z}\left(t_{0}\right) \sin t_{0}
$$

is nonnegative, because $c=0$. It follows from the assumptions. Therefore, the proof is completed.

Moreover, we also have the following results which are same as Theorem 8 and Theorem 9 in [15].

Proposition 3.7. Assume that $a>0$ and $\mu \delta \leq \frac{4}{\pi^{2}}$ a for a constant $\mu \in(0,1]$. Then, we have

$$
\lambda \geq \frac{\pi^{2}}{d^{2}}+\frac{\mu}{2}(n-1) K=\frac{\pi^{2}}{d^{2}}+\mu \alpha .
$$

Here $d$ denotes the diameter of $(M, g)$.

Proposition 3.8. Assume that $a=0$. Then, we have

$$
\lambda \geq \frac{\pi^{2}}{d^{2}}+\frac{1}{2}(n-1) K .
$$

Proof. The proofs of Propositions 3.7 and 3.8 are the same as [15]. So, we shall give just the outline of proofs for the reader's convenience (See the original paper [15] for the full details). The key point of the proofs is to choose right functions $z(t)$ in (32) and (33) enough to prove.

First, let us see the proof of Proposition 3.7. Let $\mu_{\epsilon}:=\mu-\epsilon>0$ for a small positive constant $\epsilon$. Take $b>1$ close to 1 such that $\mu_{\epsilon}<\frac{4}{\pi^{2}} c$. Let

$$
z(t):=1+c \eta(t)+\mu_{\epsilon} \delta \xi(t),
$$

where

$$
\xi(t):=\frac{\cos t^{2}+2 t \sin t \cos t+t^{2}-\frac{\pi^{2}}{4}}{\cos t^{2}} \quad \text { for } t \in\left[-\frac{\pi}{2}, \frac{\pi}{2}\right]
$$

and

$$
\eta(t):=\frac{\frac{4}{\pi} t+\frac{4}{\pi} \cos t \sin t-2 \sin t}{\cos ^{2} t} \text { for } t \in\left[-\frac{\pi}{2}, \frac{\pi}{2}\right] .
$$


The needed properties of $\xi(t)$ and $\eta(t)$ for the proofs are studied in Section 4 of [15]. By using such properties and (32), we can show

$$
Z(t) \leq z(t), \quad \text { for } t \in\left[-\sin ^{-1}(1 / b), \sin ^{-1}(1 / b)\right] .
$$

The above implies

$$
\sqrt{\lambda} \geq \frac{|\nabla t|}{\sqrt{z(t)}} \quad \text { for } t \in\left[-\sin ^{-1}(1 / b), \sin ^{-1}(1 / b)\right] .
$$

Let $q_{1}$ and $q_{2}$ be the two points in $M$ such that $\nu\left(q_{1}\right)=-1$ and $\nu\left(q_{2}\right)=1$ respectively. Let $L$ be the minimum geodesic between $q_{1}$ and $q_{2}$. Integrating the both sides of (34) along $L$ and changing variable, then we have

$$
\sqrt{\lambda} d \geq \int_{L} \frac{|\nabla t|}{\sqrt{z(t)}} d l=\int_{-\frac{\pi}{2}}^{\frac{\pi}{2}} \frac{1}{\sqrt{z(t)}} d t \geq \frac{\left(\int_{-\pi / 2}^{\pi / 2} d t\right)^{3 / 2}}{\left(\int_{-\pi / 2}^{\pi / 2} z(t) d t\right)^{1 / 2}} \geq\left(\frac{\pi^{3}}{\int_{-\pi / 2}^{\pi / 2} z(t) d t}\right)^{1 / 2} .
$$

From the properties of $\xi(t)$ and $\eta(t)$, and the definition of $z(t)$, we can show that

$$
\int_{-\pi / 2}^{\pi / 2} z(t) d t=\left(1-\mu_{\epsilon} \delta\right) \pi
$$

Hence we have

$$
\lambda \geq \frac{\pi^{2}}{\left(1-\mu_{\epsilon} \delta\right) d^{2}}
$$

From $\mu_{\epsilon}<\mu \leq 1$ and

$$
\delta=\frac{\alpha}{\lambda} \leq \frac{(n-1) K}{2} \cdot \frac{1}{(n-1) K}=\frac{1}{2} \quad(\text { due to Proposition } 3.1)
$$

we have $1-\mu_{\epsilon} \delta \geq \frac{1}{2}>0$. Then (35) implies

$$
\lambda \geq \frac{\pi^{2}}{d^{2}}+\lambda \mu_{\epsilon} \delta=\frac{\pi^{2}}{d^{2}}+\mu_{\epsilon} \alpha .
$$

Letting $\epsilon \rightarrow 0$, we have the desired inequality.

Next, let us see the proof of Proposition 3.8. In this case, the test function is defined by

$$
y(t)=1+\delta \xi(t)
$$

Then, by using (33), we can get $Z(t) \leq y(t)$ for $t \in[-\pi / 2, \pi / 2]$. The rest of the proof is similar to that of Proposition 3.7.

TheOREM 3.9. Let $(M, g, \phi)$ be an $n$-dimensional compact Bakry-Émery manifold without boundary. Assume that there exists a constant $K>0$ such that

$$
\operatorname{Ric}_{\phi} \geq(n-1) K g
$$

for all $x \in M$. Then the first non-zero eigenvalue $\lambda$ of the Bakry-Émery Laplacian $\Delta_{\phi}$ on $M$ satisfies

$$
\lambda \geq \frac{\pi^{2}}{d^{2}}+\frac{31}{100}(n-1) K \quad \text { for } n \geq 2,
$$


where $d$ denotes the diameter of $(M, g)$.

Proof. The proof is almost same as in [15], because all necessary results for the proof hold in the same way as in [15] except Proposition 3.1. The proof in [15] are proceeded separately in the following cases;

- Case (A): $a=0$.

- If $0<a<1$,

- Case (B-1): $\frac{\pi^{2}}{4} \delta \leq a$.

- If $a<\frac{\pi^{2}}{4} \delta$,

* Case (B-2-a): $0.765 \leq a$.

$*$ If $0<a<0.765$,

- Case (B-2-b1): $1.53 \delta \leq a$.

- Case (B-2-b2): $a<1.53 \delta$.

The case whose proof requires Proposition 3.1 is only Case (B-a-2): $0.765 \leq a<\frac{\pi^{2}}{4} \delta$. This means that we can prove the other cases in the same way and get the same statement as in [15]. For the reader's convenience, we shall give the outline of the proofs except Case (B-a-2). In the cases except (B-a-2) and (B-2-b2), we can prove by applying Propositions 3.7 and 3.8 directly. More precisely, we can apply
Proposition 3.8
to Case $(\mathrm{A})$
Proposition 3.7
Proposition 3.7 for $\mu=\frac{4}{\pi^{2}} \frac{a}{8}$
to Case (B-1);
to Case (B-2-b1).

Then, from Cases (A), (B-1) we can get

$$
\lambda \geq \frac{\pi^{2}}{d^{2}}+\frac{1}{2}(n-1) K .
$$

From Case (B-2-b1) we can get

$$
\lambda \geq \frac{\pi^{2}}{d^{2}}+\frac{31}{100}(n-1) K .
$$

The proof in Case (B-2-b2) is essentially similar to that of Proposition 3.7, although it is more technical than other cases. The essential part of the proof is to show $Z(t) \leq z(t)$ on $\left[-\sin ^{-1}(1 / b), \sin ^{-1}(1 / b)\right]$ with respect to a new test function

$$
z(t):=1+c \eta(t)+\left(\delta-\sigma c^{2}\right) \xi(t) \quad \text { for } t \in\left[-\sin ^{-1}(1 / b), \sin ^{-1}(1 / b)\right],
$$

where

$$
\sigma=\frac{\tau}{\left(\left[\frac{3}{2}-\frac{\pi^{2}}{8}-\left(\frac{\pi^{2}}{32}-\frac{1}{6}\right) \frac{153}{100}\right] \frac{200}{153}-\frac{\left(\frac{8}{3 \pi}-\frac{\pi}{4}\right)^{2}}{\left[-1+\left(12-\pi^{2}\right) \frac{100}{153}\right]}\right) c}>0 .
$$

Then, we can get (36).

Next, let us consider Case (B-a-2). Since $\left(\frac{4}{\pi^{2}} \frac{a}{\delta}\right) \in(0,1]$ and $\left(\frac{4}{\pi^{2}} \frac{a}{\delta}\right) \delta=\frac{4}{\pi^{2}} a$, we can apply Proposition 3.7 by putting $\mu=\left(\frac{4}{\pi^{2}} \frac{a}{\delta}\right)$. Then we have

$$
\lambda \geq \frac{\pi^{2}}{d^{2}}+\left(\frac{4}{\pi^{2}} \frac{a}{\delta}\right) \alpha=\frac{\pi^{2}}{d^{2}}+\frac{4 a}{\pi^{2}} \lambda .
$$

On the other hand, Proposition 3.1 implies

$$
\lambda \geq(n-1) K=2 \alpha .
$$


REMARK 3.10. This inequality corresponds to $\lambda \geq \frac{2 n}{n-1} \alpha$ in pp.39\%, [15].

From these two inequalities, we have

$$
\begin{aligned}
\lambda & \geq \frac{\pi^{2}}{d^{2}}+\frac{8 a}{\pi^{2}} \alpha \\
& \geq \frac{\pi^{2}}{d^{2}}+\frac{8(0.765)}{\pi^{2}} \alpha .
\end{aligned}
$$

Since

$$
\frac{8(0.765)}{\pi^{2}}>0.62=\frac{31}{50}
$$

we get

$$
\lambda \geq \frac{\pi^{2}}{d^{2}}+\frac{31}{50} \alpha=\frac{\pi^{2}}{d^{2}}+\frac{31}{100}(n-1) K,
$$

which is $(36)$.

REMARK 3.11. In [15], it is proved that we can get the better result when $n=2$ than $n \geq 3$ for ordinary Laplacian. However, since our Proposition 3.1 is weaker than Lemma 3 in [15], the same argument does not hold for Bakry-Emery Laplacian.

COROLlARY 3.12. Let $(M, g)$ be a compact nontrivial gradient shrinking soliton with $\operatorname{dim} M \geq 4$ satisfying

$$
R_{i j}-\gamma g_{i j}+\nabla_{i} \nabla_{j} f=0 .
$$

Let $\Delta_{f}$ be the corresponding Bakry-Émery Laplacian defined by

$$
\Delta_{f}=\Delta-\nabla f \cdot \nabla
$$

where $\Delta=g^{i j} \nabla_{i} \nabla_{j}$. If $-\lambda$ is the first non-zero eigenvalue of $\Delta_{f}$ then we have

$$
\lambda \geq \frac{\pi^{2}}{d^{2}}+\frac{31}{100} \gamma
$$

Proof. We have only to apply Theorem 3.9 with $\gamma=(n-1) K$.

\section{Proof of Theorem 1.2.}

Proof of Theorem 1.2. Let $(M, g)$ be a compact nontrivial gradient shrinking soliton with $\operatorname{dim} M \geq 4$ satisfying

$$
R_{i j}-\gamma g_{i j}+\nabla_{i} \nabla_{j} f=0 .
$$

Let $\Delta_{f}$ be the corresponding Bakry-Émery Laplacian defined by

$$
\Delta_{f}=\Delta-\nabla f \cdot \nabla
$$

where $\Delta=g^{i j} \nabla_{i} \nabla_{j}$. By Lemma $2.1 \Delta_{f}$ has an eigenvalue $-2 \gamma$. Thus by Corollary 3.12 we have

$$
2 \gamma \geq \frac{\pi^{2}}{d^{2}}+\frac{31}{100} \gamma
$$

from which (2) follows easily. This completes the proof of Theorem 1.2. 


\section{REFERENCES}

[1] B. ANDREW AND L. Ni, Eigenvalue comparison on Bakry-Emery manifolds, arXiv:1111.4967.

[2] H.-D. CAO, Existence of gradient Kähler-Ricci solitons, Elliptic and Parabolic Methods in Geometry (Minneapolis, MN, 1994), A. K. Peters (ed.), Wellesley, MA, 1996, pp. 1-16.

[3] H.-D. CAO, Geometry of Ricci solitons, Chinese Ann. Math. Ser. B, 27:2 (2006), pp. $121-142$.

[4] A. Derdzinski, A Myers-type theorem and compact Ricci solitons, Proc. Amer. Math. Soc, 134 (2006), pp. 3645-3648 .

[5] A. Derdzinski, Compact Ricci solitons, preprint.

[6] M. Fernández-López And E. Garcia-Rio, Diameter bounds and gap theorems for compact Ricci solitons, preprint.

[7] A. Futaki, The Ricci curvature of symplectic quotients of Fano manifolds, Tohoku Math. J. (2), 39:3 (1987), pp. 329-339.

[8] A. Futaki, H. LI, AND X.-D. Li, On the first eigenvalue of the Witten-Laplacian and the diameter of compact shrinking solitons, arXiv:1111.6364, to appear in Ann. Glob. Anal. Geom.

[9] R.S. Hamilton, The Ricci flow on surfaces, Mathematics and general relativity (Santa Cruz, CA, 1986), pp. 237-262, Contemp. Math., 71, Amer. Math. Soc., Providence, RI, 1988.

[10] R. S. Hamilton, The formation of singularities in the Ricci flow, Surveys in Differential Geometry (Cambridge, MA, 1993), 2, International Press, Combridge, MA, 1995, pp. 7136.

[11] T. IveY, Ricci solitons on compact three-manifolds, Differential Geom. Appl., 3:4 (1993), pp. 301-307.

[12] N. KoIso, On rotationally symmetric Hamilton's equation for Kähler-Einstein metrics, Recent topics in differential and analytic geometry, pp. 327-337, Adv. Stud. Pure Math., 18-I, Academic Press, Boston, MA, 1990.

[13] H. Li, Gap theorems for Kähler-Ricci solitons, Arch. Math., 91:2 (2008), pp. 187-192.

[14] P. Li AND S.-T. YAU, Estimates of eigenvalues of a compact Riemannian manifold, Geometry of the Laplace operator (Proc. Sympos. Pure Math., Univ. Hawaii, Honolulu, Hawaii, 1979), pp. 205-239, Proc. Sympos. Pure Math., XXXVI, Amer. Math. Soc., Providence, R.I., 1980

[15] J. LING Lower bounds of the eigenvalues of compact manifolds with positive Ricci curvature, Ann. Global Anal. Geom., 31:4 (2007), pp. 385-408.

[16] Z. LU AND J. Rowlette, The fundamental gap, preprint, arXiv:1003.0191v1.

[17] G. Perelman, The entropy formula for the Ricci flow and its geometric applications, http://arXiv.org/abs/math.DG/02111159, 2002.

[18] X.-J. WANG AND X. ZHU, Kähler-Ricci solitons on toric manifolds with positive first Chern class, Adv. Math., 188:1 (2004), pp. 87-103. 
A. FUTAKI AND Y. SANO 